\title{
Correction to: Drug-Induced Dental Caries: A Disproportionality Analysis Using Data from VigiBase
}

\author{
Emilie Patras de Campaigno ${ }^{1}$ (1) Inès Kebir $^{1} \cdot$ Jean-Louis Montastruc $^{1,2,3,4,5}$. \\ Manuela Rueter $^{1} \cdot$ Delphine Maret $^{6,7,8} \cdot$ Maryse Lapeyre-Mestre $^{1,2,3,4}$ (D) \\ Brigitte Sallerin $^{9,10} \cdot$ Fabien Despas ${ }^{1,2,3,4}$ (i)
}

Published online: 8 May 2018

(C) Springer International Publishing AG, part of Springer Nature 2018

\section{Correction to: Drug Saf (2017) 40(12):1249-1258 https://doi.org/10.1007/s40264-017-0575-5}

In the original publication, first author's family name and first name were incorrectly tagged. The first name and family name should read as Emilie and Patras De Campaigno, respectively.

The original article can be found online at https://doi.org/10.1007/ s40264-017-0575-5.

Fabien Despas

fabien.despas@univ-tlse3.fr

1 Service de Pharmacologie Médicale et Clinique, Centre Hospitalier Universitaire de Toulouse, 31000 Toulouse, France

2 Laboratoire de Pharmacologie Médicale et Clinique, Faculté de Médecine, Université Paul Sabatier, 37 Allée JulesGuesde, 31000 Toulouse, France

3 INSERM UMR 1027, Faculté de Médecine, Université de Toulouse, Toulouse, France

4 CIC INSERM 1436, Université et Centre Hospitalier Universitaire de Toulouse, Toulouse, France

5 Centre Midi-Pyrénées de PharmacoVigilance, de Pharmacoépidémiologie et d'Informations sur le Médicament et Pharmacopôle Midi-Pyrénées, Centre Hospitalier Universitaire de Toulouse, Toulouse, France
6 Laboratoire AMIS, CNRS, UMR 5288, 31000 Toulouse, France

7 Service Odontologie Conservatrice, Endodontie, Université de Toulouse III, 31000 Toulouse, France

8 Service Odontologie Conservatrice, CHU Toulouse, 31000 Toulouse, France

9 Service de Pharmacie Hospitalière, Centre Hospitalier Universitaire de Toulouse, 31000 Toulouse, France

10 Laboratoire de Pharmacie Clinique, Faculté de Pharmacie de Toulouse, 31000 Toulouse, France 